\title{
Sismo de San Gabriel, 27 de julio de 2018 (Ml 4,6): análisis de los parámetros de la fuente sísmica y del contexto sísmico de la región suroeste del Valle Central, Costa Rica
}

\author{
Daniela Campos-Durán $^{1^{*}}$; ; Ronnie Quintero-Quintero ${ }^{2} \mathbb{D}$; Juan Segura-Torres ${ }^{2}$
}

Forma de citar: Campos-Durán, D.; Quintero-Quintero, R.; Segura-Torres, J. (2021). Sismo de San Gabriel, 27 de julio de 2018 (Ml 4,6): análisis de los parámetros de la fuente sísmica y del contexto sísmico de la región suroeste del Valle Central, Costa Rica. Boletín de Geología, 43(3), 165-178. https://doi.org/10.18273/revbol. v43n3-2021008

\section{Resumen}

Se caracterizan y analizan los parámetros de la fuente sísmica del sismo ocurrido el 27 de julio de 2018 a las 17:50:46 (hora UTC) en San Gabriel de Aserrí (latitud 9,788, longitud -84,101). Este tuvo una profundidad de 15 km y magnitud Ml 4,6. La distribución de la sismicidad localizada y solución de mecanismo focal (MF) indican que probablemente el evento ocurrió en una falla de corrimiento lateral derecho, con componente normal pequeña y rumbo NNW, alto ángulo de buzamiento $\sim 77^{\circ}$ e inclinación hacia el NE (strike 326\% dip 77\%/rake $-165^{\circ}$ ). Este MF concuerda con otros localizados en el área, el cual evidencia deformación transtensional en el antearco central del país, conocido como el Cinturón Deformado del Centro de Costa Rica (CDCCR). Registros de aceleración en las estaciones sísmicas (\%g), permitieron identificar en la escala de Intensidad Modificada de Mercalli (IMM) intensidades instrumentales máximas de IV en comunidades como San Gabriel de Aserrí, San Pablo de León Cortés y San Marcos de Tarrazú.

Palabras clave: Costa Rica; Sismo de San Gabriel; Tensor de momento; Réplicas.

\section{San Gabriel earthquake, July 27, 2018 (Ml 4.6): seismic source parameters and the seismic context of the southwestern region of the Central Valley of Costa Rica}

\begin{abstract}
In this research the seismic source parameters of the earthquake that occurred on July 27, 2018, at 17:50:46 (UTC time) in San Gabriel de Aserrí (latitude 9.788, longitude -84.101) are characterized and analyzed. The parameters show that the event had a depth of $15 \mathrm{~km}$ and $\mathrm{Ml} \mathrm{4.6.} \mathrm{The} \mathrm{distribution} \mathrm{of} \mathrm{localized} \mathrm{seismicity}$ and a focal mechanism solution indicates that it probably occurred on a right-lateral strike-slip fault with a small normal component, strike NNW, high dip angle $\sim 77^{\circ}$, dipping to the NE (strike 326\% dip $77^{\circ} /$ slip $-165^{\circ}$ ). The focal mechanism is consistent with those of previous seismicity located in the area and they indicate transtensional deformation in the central forearc of the country, known as the Central Costa Rica Deformed Belt. The instrumental intensities, obtained from the acceleration (\% g) recorded in the seismic stations, allowed to identify in the Modified Mercalli Intensity scale (MMI) maximum instrumental intensities of IV in communities such as San Gabriel de Aserrí, San Pablo de León Cortés, and San Marcos de Tarrazú.
\end{abstract}

Keywords: Costa Rica; San Gabriel earthquake; Moment tensor; Aftershocks.

${ }^{1}$ Escuela de Ciencias Geográficas, Universidad Nacional de Costa Rica, Costa Rica. (*) daniela.campos.duran@una.cr ${ }^{2}$ Observatorio Vulcanológico y Sismológico de Costa Rica, Universidad Nacional de Costa Rica, Costa Rica. ronnie.quintero.quintero@una.cr; jusegura1958@gmail.com 


\section{Introducción}

El estudio de los sismos someros $(\leq 25 \mathrm{~km})$ en la región central de Costa Rica ha brindado mayor comprensión acerca del comportamiento de las fuentes sísmicas ubicadas en esta área, donde los fallamientos superficiales han generado en la historia reciente eventos importantes. Entre los que destacan el terremoto de Patillos del 30 de diciembre de 1952 (Ms 5,9 según Montero y Alvarado, 1995), el de Piedras Negras del 22 de diciembre de 1990 ( $M w$ 6,0), el de Cinchona del 08 de enero de 2009 (Mw 6,1) y el más reciente el de Capellades del 1 de diciembre de 2016 (Mw 5,4) (Campos-Durán y Quintero-Quintero, 2020).

Tectónicamente, Costa Rica se caracteriza por la subducción de la placa del Coco (CO) bajo la placa Caribe (CA), donde la velocidad de convergencia es de 7,9 cm/año con dirección NE (UNAVCO, 2020) (Figura 1). La interacción CO-CA genera en la placa continental fallamientos superficiales activos (Marshall et al., 2000), los que han dado lugar a sismicidad al SW del Valle Central, tal como el evento en estudio, denominado en esta investigación sismo de San Gabriel.

Se destacan en esta área varias fallas mapeadas, tales como La Candelaria, Picagres, San Antonio, Jaris, Jericó, Frailes, Aserrí, Escazú y Belo Horizonte y otras de menor longitud que a la fecha no han sido caracterizadas. El monitoreo permanente señala una importante actividad sísmica, lo que podría contribuir a la identificación del trazo de estructuras pequeñas en superficie (Figura 1).

En esta investigación se caracterizan y analizan los parámetros de la fuente sísmica y las intensidades instrumentales en la escala de IMM del evento superficial de baja magnitud (Ml 4,6), sismo de San Gabriel, ocurrido el 27 de julio de 2018, a las 17:50:46 (hora UTC), en el antearco central de Costa Rica, con epicentro $16 \mathrm{~km}$ al SW de la ciudad de San José (Figura 1).

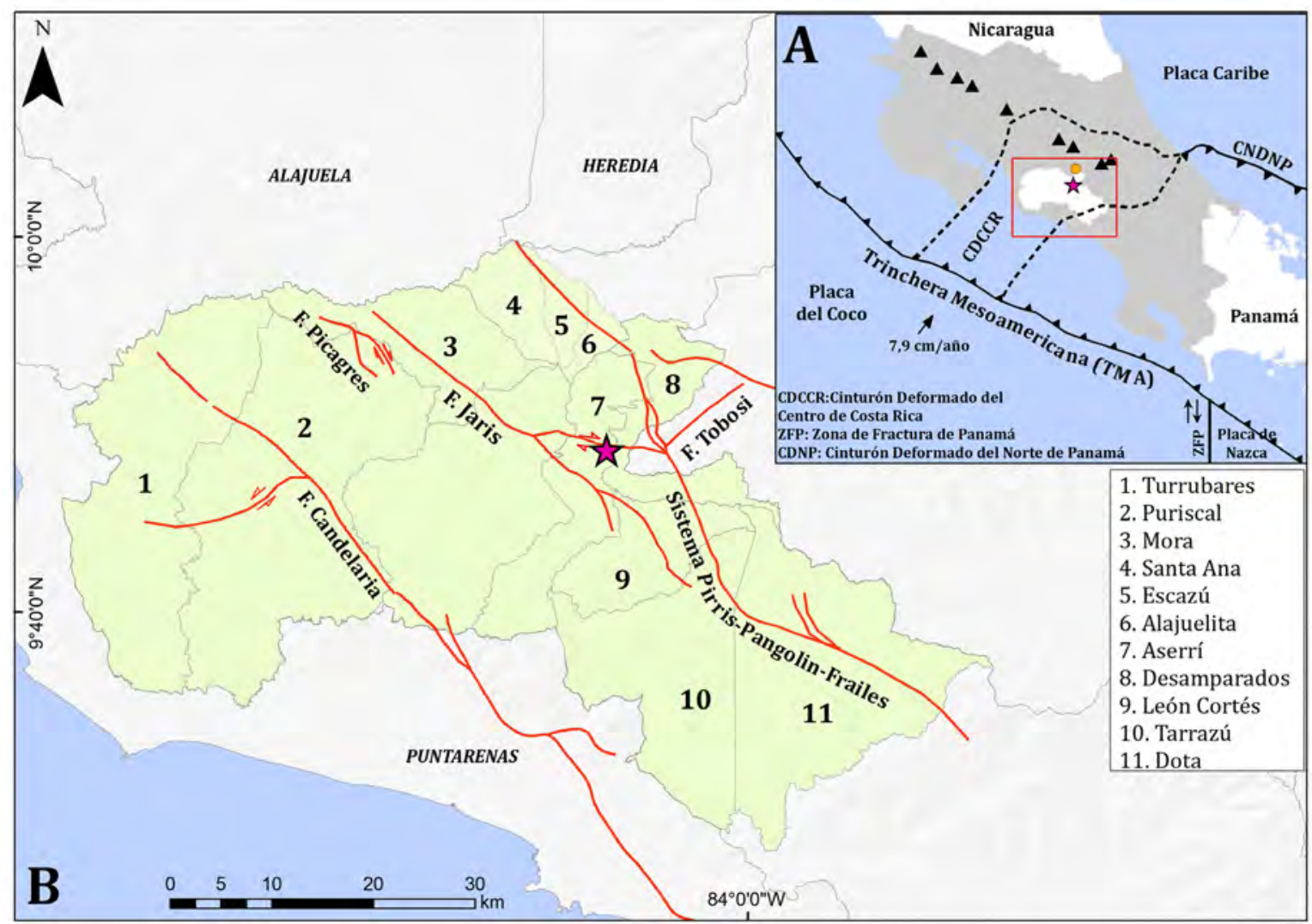

Figura 1. A. Localización del epicentro del sismo de San Gabriel (estrella fucsia), los volcanes se señalan con triángulos negros y la capital San José con un círculo naranja. Las líneas punteadas al interior de la región central del país delimitan el CDCCR, la zona de acople entre las placas del Coco y Caribe se representan con una línea negra con triángulos a lo largo de la Trinchera Mesoamericana (TM) B. Área de estudio, región SW del Valle Central de Costa Rica y principales fallas tectónicas identificadas por Arias y Denyer (1991), Montero (1999), Denyer et al. (2009) y Montero y Rojas (2014). Estas estructuras atraviesan 12 cantones, de los cuales, Desamparados, Alajuelita, Escazú y Santa Ana corresponden a centros urbanos. 
Se utilizaron datos de las estaciones sísmicas del Observatorio Vulcanológico y Sismológico de Costa Rica (OVSICORI-UNA), de aquí en adelante llamada red principal, así como de la red de Nicaragua (INETER), GEOSCOPE, de la Red Sismológica Nacional (RSNUCR) y del Laboratorio de Ingeniería Sísmica de la Universidad de Costa Rica (LIS-UCR).

Los datos de las estaciones sísmicas de la red principal permitieron la lectura de los arribos de las ondas $\mathrm{P}$ y S y la relocalización de la sismicidad. Además, se realizó la inversión de la forma de onda cerca del hipocentro para la ubicación del centroide; escogiendo el que presentó mayor correlación entre la forma de onda real y la sintética.

Aunque el sismo en estudio no generó daños de consideración, fue sentido y reportado como moderado en San Gabriel de Aserrí, San Pablo de León Cortés y San Marcos de Tarrazú. En otras comunidades como Curridabat, San José centro, La Guácima de Alajuela, Guadalupe, Heredia centro y San Pedro de Montes de Oca fue percibido como leve. Para determinar la escala IMM se implementaron las intensidades instrumentales registradas en las estaciones sísmicas, utilizando como parámetro el valor de aceleración (\%g), donde se obtuvieron valores entre I y IV.

Desde la instalación de la red principal en 1984 se tiene registro de importante sismicidad superficial en todo el país, siendo más prominente en la región central, desde el antearco hasta el arco volcánico y menor en la costa Caribe (Figura 2). En esta área QuinteroQuintero y Porras-Espinoza (2018) encontraron que los sismos con magnitud moderada o fuerte presentan una recurrencia estimada de 21 años.

Es a partir de 1989 que se recopila y documenta información sobre sismos sentidos, considerando al menos los siguientes criterios: a) haber sido reportado por la población o b) registrar daños estructurales. Con estos parámetros destacan en el área de estudio el evento de Alto El Aguacate de 1989 (Mw 5,3), el de Puriscal del 30 de junio de 1990 (Mw 5,5), el de Piedras Negras del 22 de diciembre de 1990 (Mw 6,0), el de Frailes del 9 de agosto de 1991 (Mw 5,2) y el del 7 de agosto del 2014 (Ml 4,9) $10 \mathrm{~km}$ al NW de San Marcos de Tarrazú.

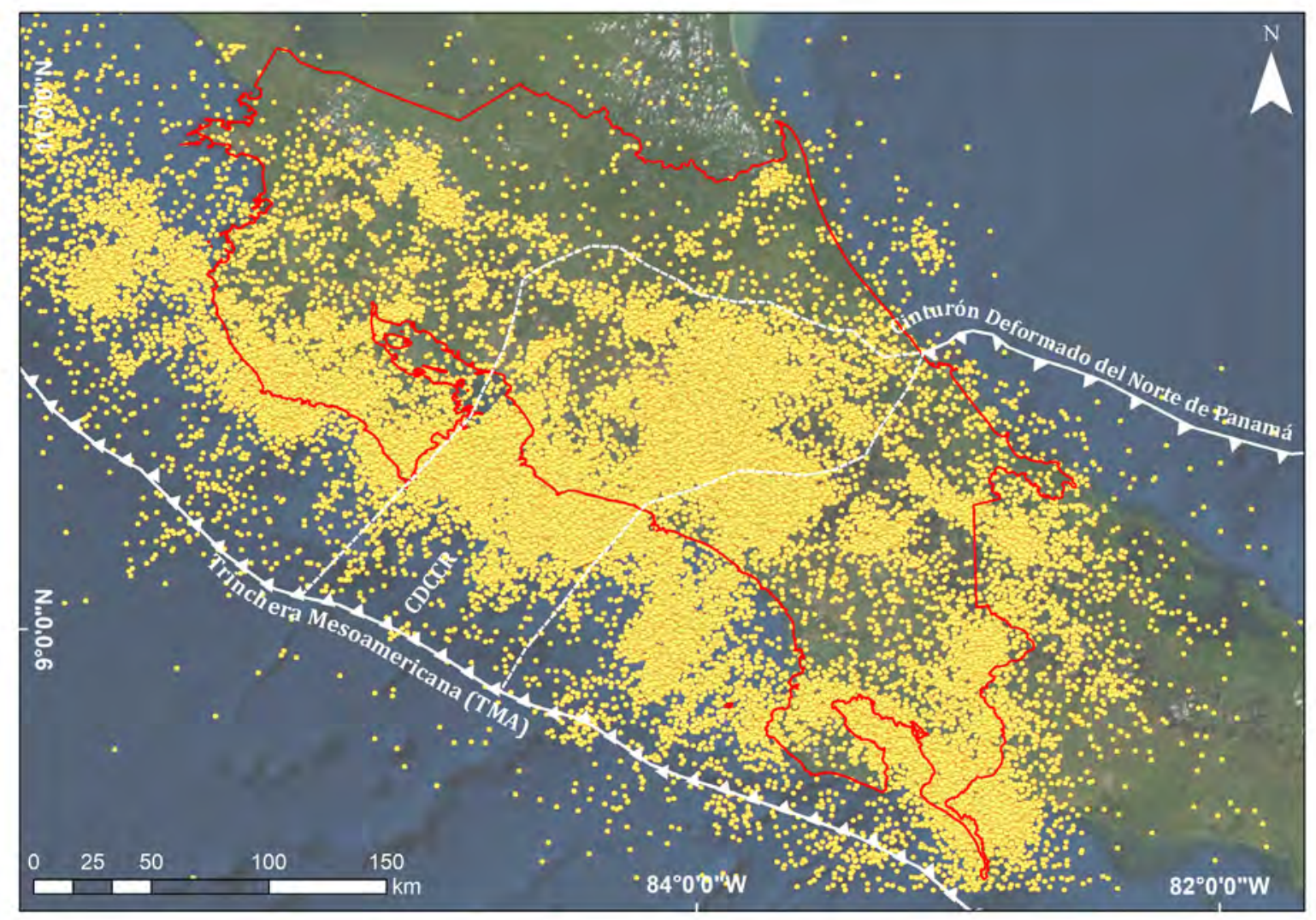

Figura 2. Sismicidad superficial (círculos amarillos) con profundidad $\leq 25 \mathrm{~km}$, registrada entre 2010-2018 por la red sísmica del OVSICORI-UNA. Se observa la concentración en la región central del país. 


\section{Metodología}

La caracterización de los parámetros sísmicos y la distribución hipocentral se obtuvo utilizando datos de 67 estaciones de la red principal. Además, se incorporaron datos complementarios de estaciones de la red de Nicaragua (ACON, BLUN), de la Red Sismográfica Global (BCIP, JTS), de GEOSCOPE (HDC), de la RSN-UCR (CVTQ, CVTR, RAFA) y del LIS-UCR (BJL1, SBEB, SESC, SGTS). Estos se recolectaron $\mathrm{y}$ almacenaron por medio del paquete ANTELOPE (http://www.brtt.com/software.html), en la base de datos sísmica del OVSICORI-UNA.

Las formas de onda de la sismicidad del 2018, almacenadas en la base antes citada, permitió la lectura de las llegadas de las ondas P y S, así como su relocalización, seleccionando los arribos de la onda $\mathrm{S}$ en componentes horizontales. Además, se enfatizó particularmente en la calidad del arribo y polaridad de algunos sismos para el cálculo del MF usando el programa FOCMEC que está integrado dentro del paquete SeiSan (Havskov y Ottemoller, 1999).

Los hipocentros se calcularon utilizando las librerías GenLoc (Fan et al., 2006), programa que es parte del paquete ANTELOPE, además se implementó el modelo de velocidad de Quintero y Kissling (2001).

Una vez relocalizado el evento principal, se utilizó la ubicación y formas de onda de velocidad y aceleración de nueve estaciones sísmicas alrededor del epicentro (OCM, HDC3, PEDER, RIMA, VTLA, CDM, CPMI, JACO, PEZE) para estimar el Tensor de Momento del Centroide (CMT).

Las estaciones se localizaron entre 19 y $65 \mathrm{~km}$ del epicentro y consisten en instrumentos STS2.0 con digitalizador Q330HR (CMD, JACO), Nanometric 240 con digitalizador Quanterra 330HR (CPMI, PEZE), Guralp CMG3ESPC con digitalizador DM24-MK3 (VTLA), Trillium Compact 120 con digitalizador Taurus (PEDER) y acelerómetro FBA ES-T con digitalizador Q330HR (OCM, RIMA, HDC3).

La inversión completa de la forma de onda se realizó con el paquete ISOLA(Sokos y Zahradnik, 2008, 2013), mediante una inversión debajo del epicentro y después en un plano horizontal para encontrar el centroide; la cual optimiza la correlación entre la forma de onda real y la sintética.

Por ser un sismo de baja magnitud, para el cálculo del centroide se combinaron datos de estaciones ubicadas entre 1 y 5 veces la profundidad hipocentral, se optó por aplicar el método de las envolventes (Zahradnik y Sokos, 2018; Carvalho et al., 2019) para corroborar el MF obtenido usando inversión de las formas de onda, ya que esta técnica permite calcularlo aunque no se conozca con exactitud el modelo de velocidad real, así como reducir algunos problemas en la orientación de los sismómetros y polaridades invertidas (Zahradnik y Sokos, 2018).

También se comparó el resultado de ISOLA con el obtenido usando polaridades de los primeros impulsos, lo que permitió conocer sus calidades en las diferentes estaciones sísmicas de la red para ser usadas en el cálculo del MF de otros sismos de menor magnitud que cuenten con suficientes datos o arribos.

La intensidad instrumental en la escala de IMM se obtuvo por medio del paquete SHAKEMAP (Wald et al., 2006) que utiliza los datos hipocentrales, la magnitud, la aceleración y la velocidad pico en cada estación sísmica para estimar la intensidad instrumental.

Además, se tabularon las aceleraciones pico en distintas estaciones sísmicas como porcentaje de la gravedad (\%g) (Tabla 1). La cartografía fue generada con el software GMT de Wessel et al. (2019).

En relación con la información histórica de la sismicidad superficial registrada a partir de 1989, se realizó la búsqueda y sistematización de artículos científicos e informes técnicos referentes a eventos con una magnitud mayor a Ml 4,0. Entre los que presentaron estas características sobresalen: a) el del 26 de febrero de 1989, conocido como el del Alto El Aguacate (Mw 5,3), b) el de Frailes del 9 de agosto de 1991 (Mw 5,2) y c) y el del 7 de agosto del 2014 (Ml 4,9) ubicado a $10 \mathrm{~km}$ al NW de San Carlos de Tarrazú.

\section{Resultados}

\section{Localización de premonitores y réplicas del sismo de San Gabriel}

El sismo principal fue localizado con la implementación de lecturas de arribos 60 P- y 31 S- con estaciones sísmicas ubicadas a una distancia epicentral entre 12 y $269 \mathrm{~km}$ y un gap azimutal de $96^{\circ}$. Solamente nueve de estas se encontraron en un rango inferior a $30 \mathrm{~km}$. Se obtuvo un hipocentro con coordenadas latitud 9,788 y longitud -84,1014, una profundidad de $15 \mathrm{~km}$ y Ml 4,6. 
Tabla 1. Intensidades instrumentales en la escala de IMM.

\begin{tabular}{ccccc}
\hline $\begin{array}{c}\text { Intensidad instrumental } \\
\text { (IMM) }\end{array}$ & Aceleración $\mathbf{( \% g )}$ & Velocidad $\mathbf{( c m} / \mathbf{s})$ & \multirow{2}{*}{ Sacudida percibida } & Potencial de daño \\
\hline I & $<0,17$ & $<0,1$ & No sentida & Ninguno \\
II-III & $0,17-1,4$ & $0,1-1,1$ & Débil & Ninguno \\
IV & $1,4-3,9$ & $1,1-3,4$ & Ligera & Ninguno \\
V & $3,9-9,2$ & $3,4-8,1$ & Moderada & Muy ligero \\
VI & $9,2-18$ & $8,1-16$ & Fuerte & Ligero \\
VII & $18-34$ & $16-31$ & Muy fuerte & Moderado \\
VIII & $34-65$ & $31-60$ & Severa & Moderado a fuerte \\
XI & $65-124$ & $60-116$ & Violenta & Fuerte \\
X + & $>124$ & $>116$ & Extrema & Muy fuerte \\
\hline
\end{tabular}

El sismo de San Gabriel fue precedido por 10 premonitores, cuya magnitud varió entre 0,9 y 3,9 Ml. Se destaca este último localizado $1 \mathrm{~km}$ al $\mathrm{NE}$ del de $\mathrm{Ml}$ 4,6, a las 15:33:20 (hora UTC) y a una profundidad de 14 km. Importante hacer hincapié en que la distribución hipocentral de estos 10 eventos tuvo el mismo patrón que la sismicidad registrada posterior al de Ml 4,6.
Se localizaron 10 réplicas en los primeros cinco días posteriores y estas muestran un alineamiento a lo largo de $14 \mathrm{~km}$ en dirección $\mathrm{N} 10^{\circ}-45^{\circ} \mathrm{W}$, con ubicación predominante al SSE del evento principal y a profundidades entre 8 y 15 km (Figura 3).
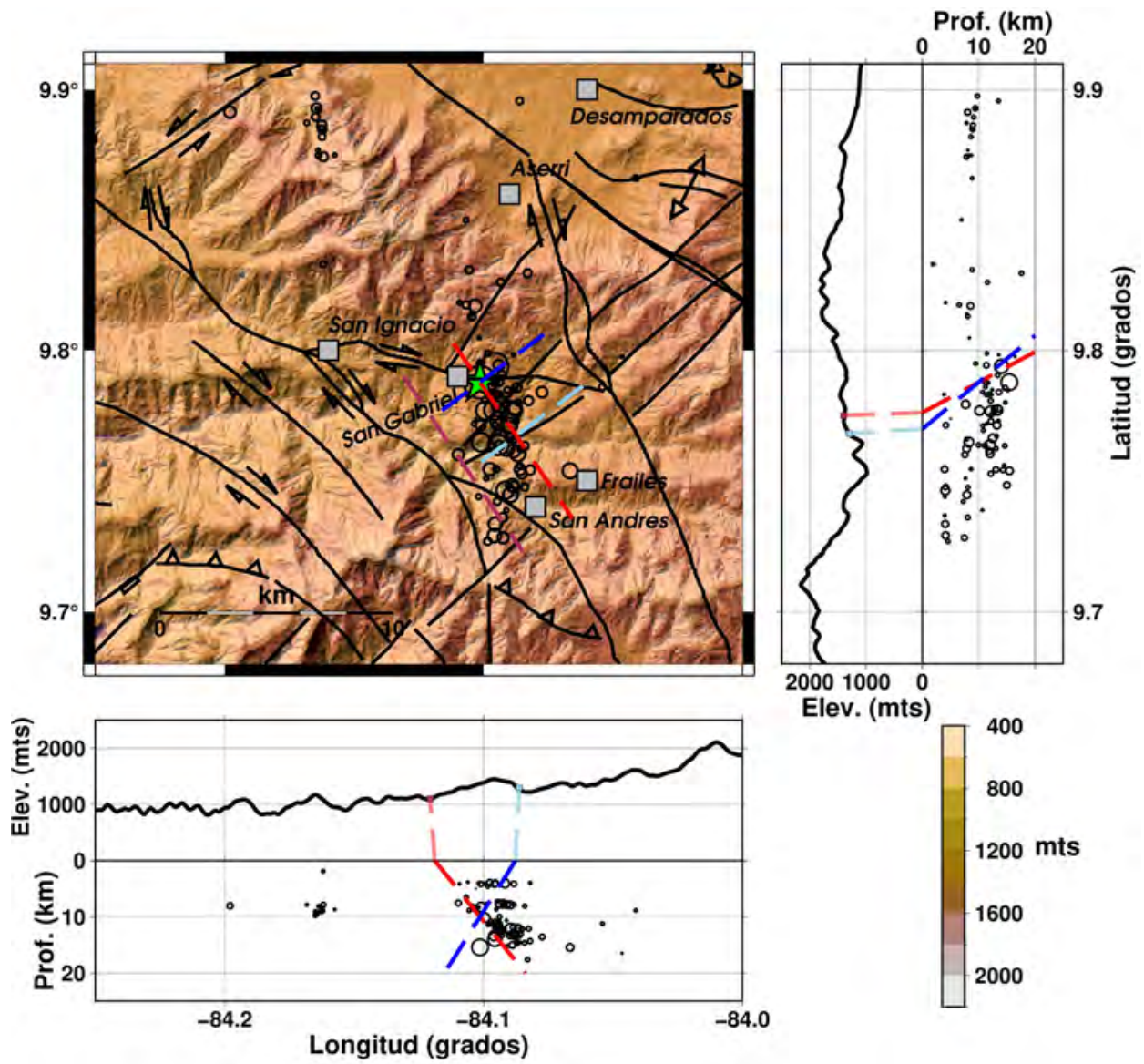

Elev. (mts)

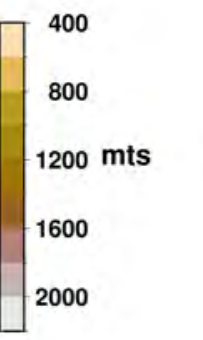

Figura 3. Sismos superficiales, asociados al evento de San Gabriel (estrella verde), localizada entre el 20 de junio y el 1 de agosto de 2018. Los epicentros están indicados por circunferencias de color negro y su tamaño de acuerdo con su magnitud Ml (entre 0,0 y 4,6). Las líneas negras muestran las fallas inferidas o mapeadas por Montero (1999), Rojas et al. (1998), Fernández y Rojas (2001) y Denyer et al. (2009). Las líneas punteadas exponen los planos nodales del evento principal, en profundidad (rojo y azul) y a 1 km de profundidad (marrón y celeste). Algunos centros de población, con su nombre respectivo, se denotan por cuadros grises. Se muestran los perfiles laterales con la sismicidad proyectada en cada uno de estos; los planos nodales con respecto al centroide se grafican en los perfiles por medio de líneas con trazos. Se expone la elevación en forma magnificada (en metros) y también la proyección de los planos nodales en superficie. Para los perfiles de elevación se tomaron los que pasan por el epicentro con dirección E-W y N-S. Elev. = elevación, Prof. = profundidad. 


\section{Mecanismo Focal}

El MF (hemisferio inferior) obtenido usando el impulso de los primeros arribos para el premonitor Ml 3,9, ocurrido el mismo día que el sismo de San Gabriel, muestra que la ruptura pudo ocurrir en un plano casi vertical con rumbo $\mathrm{N} 39^{\circ} \mathrm{W}$ que corresponde a un fallamiento de corrimiento lateral derecho (strike $321^{\circ}$ dip $86^{\circ} /$ rake $155^{\circ}$ ). El otro plano nodal tiene dirección $\mathrm{N} 53^{\circ} \mathrm{E}$, buzando $65^{\circ}$ al SE, representando un corrimiento lateral izquierdo (strike 53\%/dip 65\%/rake $5^{\circ}$ ). Los ejes de presión y tensión tienen dirección NNE y EW, respectivamente.

La solución focal para el sismo de San Gabriel con 46 polaridades (Figura 4) indica un plano nodal con parámetros de strike $327^{\circ} /$ dip $70^{\circ} /$ rake $-167^{\circ}$, eje de tensión horizontal en sentido NWW-SEE (T 280\% $6^{\circ}$ ) y eje de presión P $8^{\circ} / 23^{\circ}$ con dirección NNE (valores P y T se calcularon a partir de Zimmermann, 2009), que se asocia a una falla transcurrente dextral con componente normal y rumbo $\mathrm{N} 33^{\circ} \mathrm{W}$, inclinado $70^{\circ}$ al $\mathrm{NE}$.

Sismo de San Gobriel 27/07/2018 17:50:46

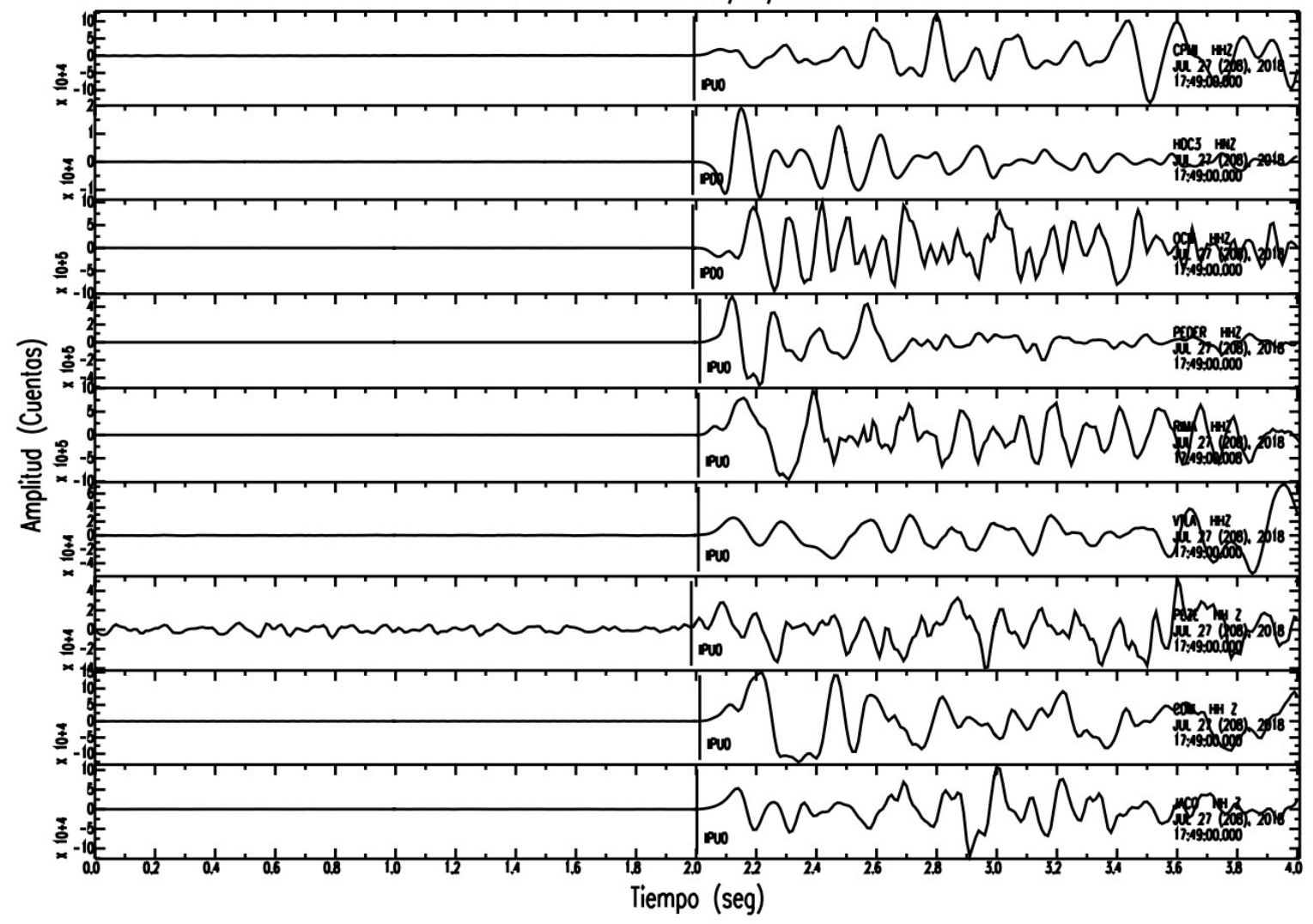

Figura 4. Formas de onda y componente vertical de algunas estaciones que registraron el sismo de San Gabriel. Se indica el arribo de la onda $\mathrm{P}$ y la polaridad.

Para el cálculo del CMT se utilizó la forma de onda de varias estaciones y el paquete ISOLA. Se probaron diferentes combinaciones en diversas direcciones desde el epicentro. Para las más cercanas se usaron datos de aceleración (OCM, HDC3, RIMA), con excepción de PEDER, que solo cuenta con instrumento de velocidad en el sitio.

Se seleccionaron formas de onda de nueve estaciones, no se usaron en la inversión aquellas con perturbación en las componentes. También se aplicaron distintos filtros con frecuencia $\mathrm{f}$, escogiendo $0,04<\mathrm{f}<0,09$
$\mathrm{Hz}$ para las estaciones VTLA, CDM, CPMI, JACO, PEZE; 0,04 $<\mathrm{f}<0,12 \mathrm{~Hz}$ para PEDER y $0,12<\mathrm{f}<0,2$ $\mathrm{Hz}$ para HDC3, OCM y RIMA, lo que dio una buena relación señal/ruido.

Los resultados de correlación entre la forma de onda real y sintética indican un centroide ubicado encima del hipocentro a $9,5 \mathrm{~km}$ de profundidad y 0,8 segundos después del inicio de la ruptura.

Se observa que la diferencia entre el coeficiente de correlación para formas de onda reales y sintéticas 
con profundidades ubicadas entre 7,5 y $13,5 \mathrm{~km}$ sobre el hipocentro no son significativas, por lo que el centroide puede ser ubicado en cualquier parte de ese rango de profundidad. Además, existe un desajuste en la amplitud entre formas de onda reales y sintéticas para las estaciones JACO y PEZE. En la componente EW de esta última se detectó cierto desajuste en el tiempo (Figura 5A).

Una de las fallas obtenidas con la inversión de formas de onda muestra un plano con strike 326\% dip 77\%/rake $-165^{\circ}$, con un porcentaje de par acoplado del $93 \%, \mathrm{Mw}$ 3,8 y una alta correlación entre las formas de onda reales y sintéticas, con una reducción de la varianza global VR=0,8 (Figura 5B).
Los resultados para diferentes profundidades fueron estables e indican un mecanismo de cizalla con pequeña componente normal. Los planos de falla tienen un alto ángulo de buzamiento, casi vertical con dirección NW con una cinemática lateral derecha y el otro hacia el SW con corrimiento lateral izquierdo.

Tanto los MF obtenidos a partir de polaridades de los primeros impulsos como los de inversión de formas de onda, muestran una estabilidad de las soluciones obtenidas usando diferentes combinaciones de estaciones. Por ejemplo, cuando se utilizan solo las más cercanas (HDC3, OCM, PEDER, RIMA) y en otro caso cuando se incorporan las más lejanas al epicentro (CPMI, JACO y PEZE).

\section{A Fecha y hora de evento: 27/07/2018- 17:50:21.00 Banda inversión (Hz): 0.04-0.2}
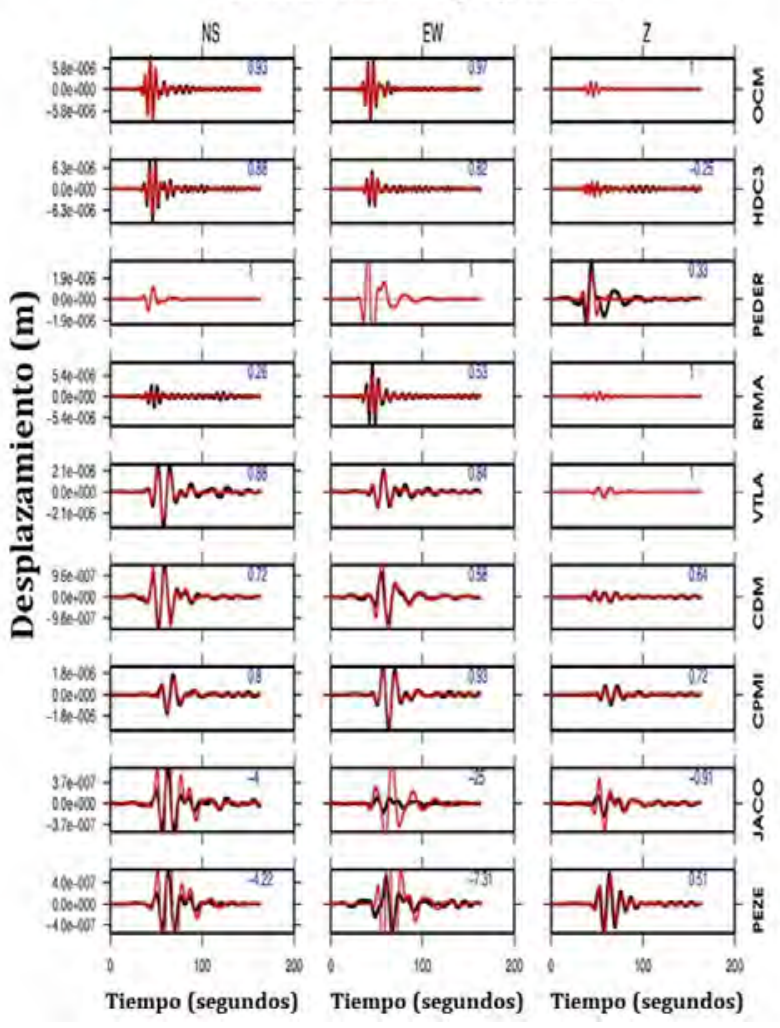

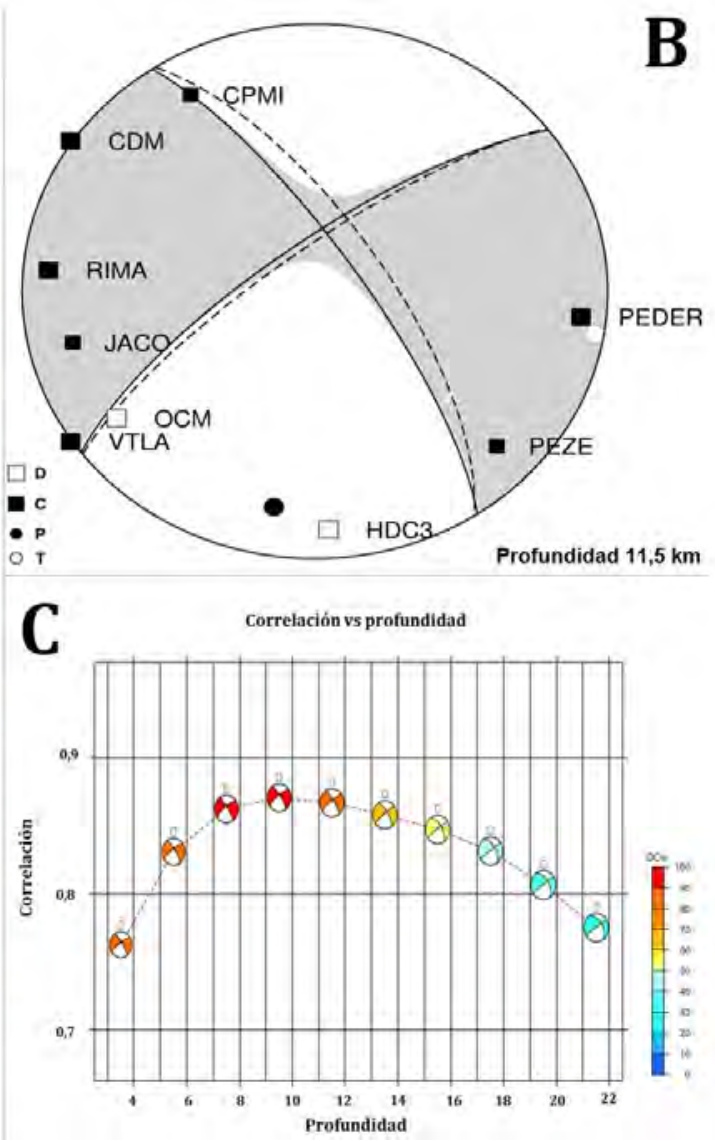

Figura 5. A. Correlación de las formas de onda de desplazamiento reales (negro) y sintéticas (gris) para el sismo de San Gabriel, filtradas en un ancho de banda de 0,04-0,2 Hz. La nomenclatura de las estaciones se muestra al lado derecho. En cada cuadro se indica la reducción de la varianza, donde las componentes con valor igual a uno no se usaron en esta inversión. B. MF derivado de la polaridad de los primeros impulsos (línea discontinua) e inversión de formas de onda (línea continua). Las estaciones usadas en la inversión de esta última muestran la respectiva polaridad. Los rectángulos blancos representan la dilatación (D), los negros la compresión (C) y el circulo negro el eje de Presión (P) con dirección NNE y el blanco el de tensión (T). La esfera focal se graficó usando una profundidad de $11,5 \mathrm{~km}$. C. Mecanismos focales obtenidos a diferentes profundidades, debajo del epicentro relacionado con el coeficiente de correlación entre las formas de onda de datos reales y sintéticas. La búsqueda se hizo cada $2 \mathrm{~km}$, para 10 posiciones, comenzando a 7,5 km. Esta fue arbitraria para evitar discontinuidades del modelo de velocidad. A la derecha del gráfico se expone el porcentaje del doble par de fuerzas. 
Para verificar la solución de las formas de onda, se realizó la inversión con el uso de las envolventes de las componentes para descartar cualquier problema asociado a la polaridad de las estaciones; así como el conocimiento detallado del modelo de velocidad y cualquier error existente en la orientación de los instrumentos. Zahradnik y Sokos (2018) indican que estos aspectos se pueden presentar al calcular el MF usando formas de onda y que podrían ser solventados mediante inversión de las envolventes.

Los epicentros de los sismos localizados entre el 27 y el 30 de julio del 2018 (Figura 3) muestran un alineamiento con dirección $\sim \mathrm{N} 10^{\circ} \mathrm{W}$ que no coincide plenamente con el rumbo de los planos nodales del mecanismo obtenido por inversión de ondas sísmicas $\left(326^{\circ}\right.$ y $\left.233^{\circ}\right)$. Sin embargo, la ubicación de las réplicas del de Ml 4,6 define un plano de falla con mayor coincidencia al que tiene rumbo $\mathrm{N} 34^{\circ} \mathrm{W}$ y no al de $\mathrm{S} 53^{\circ} \mathrm{W}$, lo que es evidente en el perfil E-W. Por esta razón se considera que la estructura que originó el evento de San Gabriel se ubica al SW del poblado homónimo, con rumbo $\mathrm{N} 34^{\circ} \mathrm{W}$, buzamiento al NE con ángulo de $\sim 77^{\circ}$, con una pequeña componente normal.

Los planos de falla en superficie con rumbo NNW o SW (Figura 3) no coinciden con la estructura de Frailes, ubicada al E de los premonitores y réplicas del sismo de San Gabriel. Caso contrario la de Jaris, ya que la actividad cerca de su extremo SE y la solución del MF, sugieren que posiblemente sea esta la responsable de la sismicidad relacionada a dicho evento.

\section{Intensidad instrumental en la Escala de Intensidad Modificada de Mercalli (IMM)}

En la zona epicentral la intensidad instrumental máxima en la escala IMM fue de IV, con un PGA máximo de 5\%g. En Ochomogo $(20 \mathrm{~km}$ al $\mathrm{N}$ del evento $\mathrm{Ml} 4,6$ ) esta fue de $2,34 \% \mathrm{~g}$ y la velocidad pico en la estación más cercana (12 km del epicentro) de 1,8 $\mathrm{cm} / \mathrm{s}$; correspondiendo a una intensidad moderada. En otros centros provinciales del Valle Central, no causó mayor daño, solamente se reportó como sentido por la población (Tabla 2, Figura 6).

Con base en los reportes a través de la red social Facebook del OVSICORI-UNA, se determinó que el sismo fue sentido fuerte en San Gabriel de Aserrí, San Pablo de León Cortes, San Ignacio de Acosta, San Marcos de Tarrazú, Desamparados y Cartago; en Heredia y Alajuela se reportó como leve, lo que de acuerdo con la escala de IMM las intensidades instrumentales oscilaron entre I y IV. Importante resaltar que no se registraron daños en infraestructuras ni caída de objetos.

Tabla 2. Intensidad instrumental en la escala IMM

\begin{tabular}{ccccc}
\hline \multirow{2}{*}{ Estación } & $\mathbf{E}(\mathbf{\% g})$ & $\mathbf{N}(\mathbf{\% g})$ & $\mathbf{Z} \mathbf{( \% g )}$ & Intensidad \\
\cline { 2 - 5 } & Valor máximo & Valor máximo & Valor máximo & I-XII \\
\hline BATAN & 0,15 & 0,14 & 0,07 & I \\
CDM & 0,12 & 0,14 & 0,08 & I \\
COVE & 0,05 & 0,05 & 0,03 & I \\
CPMI & 0,15 & 0,17 & 0,11 & I \\
CTCR & 0,12 & 0,13 & 0,07 & I \\
DUNO & 0,05 & 0,06 & 0,03 & I \\
HDC3 & 0,37 & 0,18 & 0,46 & II \\
JACO & 0,14 & 0,07 & 0,09 & I \\
OCM & 2,26 & 2,34 & 1,57 & IV \\
ORTG & 0,01 & 0,01 & 0,01 & I \\
PEZE & 0,07 & 0,18 & 0,07 & I \\
POTG & 0,15 & 0,22 & 0,07 & I \\
RIMA & 0,57 & 0,77 & 0,34 & II \\
RIOS & 0,06 & 0,11 & 0,05 & I \\
TIGR & 0,12 & 0,13 & 0,04 & I \\
VMAR & 0,03 & 0,04 & 0,04 & I \\
\hline
\end{tabular}




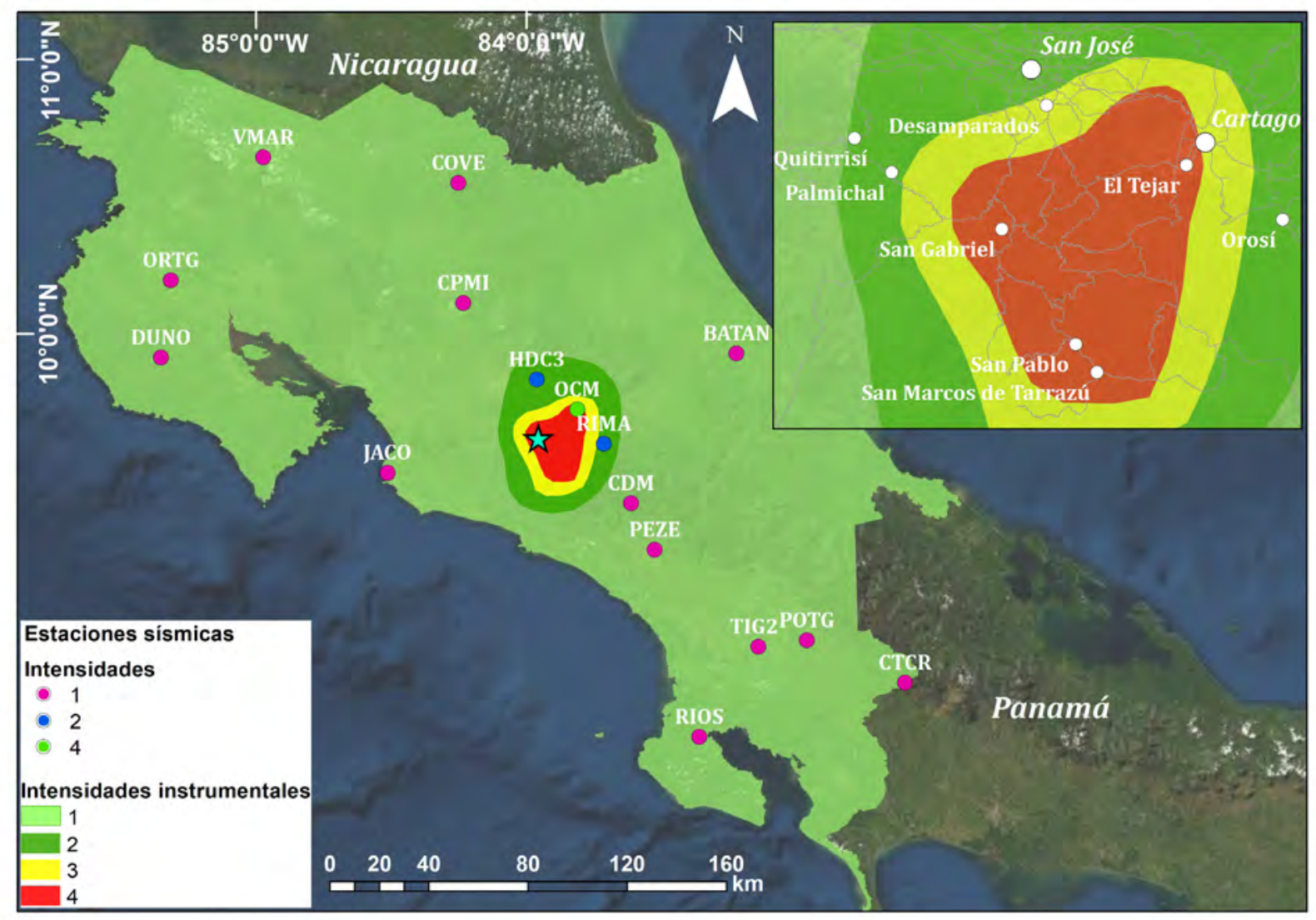

Figura 6. Isosistas en la escala IMM. Se muestran las estaciones sísmicas con círculos en color, dependiendo de la intensidad instrumental registrada en esta. El recuadro superior derecho expone un acercamiento del área donde se contabilizaron intensidades entre IV y II, siendo comunidades como San Gabriel, San Pablo y San Marcos de Tarrazú las de mayor impacto. La estrella turquesa corresponde al epicentro del sismo $\mathrm{Ml} 4,6$.

Caracterización de la sismicidad histórica en la región $S W$ del Valle Central de Costa Rica a partir de 1989

Uno de los sismos más significativos ocurrió el 26 de febrero de 1989 a las 12:21:11 (hora UTC) en Alto El Aguacate (latitud 9,665, longitud -84,183), Mw 5,3 (Ml 4,4) y profundidad de 21,5 km (Figura 7). En San Marcos de Tarrazú, San Pablo de León Cortés y Guaitil de Acosta se registraron en la escala IMM intensidades de VI y en San José y Cartago de V (Güendel, 1989).

Una solución del MF para este evento, calculado usando polaridades de los primeros impulsos, indica un plano nodal de corrimiento lateral izquierdo puro, con rumbo NEE-SWW y buzamiento vertical, strike 67\% dip 90\% rake $0^{\circ}$. Güendel (1989) consideró que este era el plano de falla y acorde con la localización de la sismicidad asociada infirió que la ruptura fue bidireccional. Resultado que presenta similitud con el reportado por el GCMT (2019), cuyos valores strike 251\% dip 80\% rake $6^{\circ}$. El segundo plano nodal asociado a este sismo y calculado con polaridades tiene parámetros strike 337\% dip 90\%/rake 180 .

Por otra parte, el análisis de los MF de las réplicas señala que los ejes de presión se orientaron NNE y los ejes de tensión ESE; ambos parámetros del sismo principal están dados por los valores P $\left(202^{\circ} / 0^{\circ}\right)$ y T $\left(112^{\circ} / 0^{\circ}\right)$, respectivamente.

Barquero et al. (1989), localizan el sismo $20 \mathrm{~km}$ al SW de San Gabriel de Aserrí y 6 km al W de la ubicación encontrada por Güendel (1989). Los autores plantean que la solución focal y la distribución de los epicentros permiten postular como probable fuente de este evento a la falla Pirris con parámetros rumbo $110^{\circ}$ y buzamiento $65^{\circ}$ (tipo normal dextral) y eje de presión con dirección NO y tensión SO; opuestos a los encontrados por Güendel (1989) y GCMT (2019). 
Para comprobar el hipocentro y MF asociado al sismo en Alto El Aguacate, se unieron datos de las red principal y de la RSN-UCR, ubicándolo 3 km al SW de la posición de Güendel (1989) o 18,5 km de San Gabriel de Aserrí, con $20 \mathrm{~km}$ de profundidad y con un plano nodal de parámetros strike $238^{\circ}$ /dip $73^{\circ}$ /rake $-10^{\circ}$ y ejes de presión $\left(15,91 \%-19,29^{\circ}\right)$ y tensión $\left(284,13^{\circ} /-5,08^{\circ}\right)$ con dirección NNE y WWN y MF de corrimiento lateral con pequeña componente normal; lo cual lo acerca obtenido en el catálogo global (GCMT, 2019).

El 9 de agosto de 1991 se registró el sismo de Frailes a las 09:33:49 (hora UTC), $7 \mathrm{~km}$ al SE del evento de San Gabriel, profundidad de $10 \mathrm{~km}$ y magnitud Mw 5,2 (Ml 4,6). De acuerdo con el mecanismo GCMT (2019) este fue de corrimiento lateral con parámetros strike 158\% dip $90^{\circ} /$ rake $-180^{\circ}$. Según Montero et al. (2016), se encuentra asociado con la parte W de la falla Navarro, la cual presenta rumbo SW-NE (Figura 7).

Este registró una IMM máxima de VII, generando daños en aproximadamente 40 viviendas. El área afectada fue alrededor de $50 \mathrm{~km}^{2}$ y los núcleos poblacionales más impactados se ubicaron en Frailes y San Cristóbal de Desamparados, en Santa Cruz de León Cortés y en Corralillo y Llano Grande de Cartago (RSN-UCR, 2015).

El 7 de agosto de 2014 tuvo lugar otro evento, a las 08:50:03 (hora UTC), $20 \mathrm{~km}$ al W de San Marcos de Tarrazú (latitud 9,652 y longitud -84,1985), con profundidad de 22,6 km y Ml 4,9. Una solución del MF usando polaridades de primeros impulsos indica un plano de falla con strike $221^{\circ} /$ dip $61^{\circ} /$ rake $-41^{\circ}$, lo que sugiere un sismo normal con el eje P semivertical y el eje $\mathrm{T}$ horizontal con dirección E-W.

Los datos utilizados de la RSN-UCR (2014), señalan que fue percibido como fuerte en el Valle Central, así como en la Zona de los Santos, Pérez Zeledón, Naranjo, San Ramón y Puriscal, las intensidades en la Escala IMM registró valores máximos de III.

Un resumen de los parámetros sísmicos de los eventos citados anteriormente se muestra en la Tabla 3.

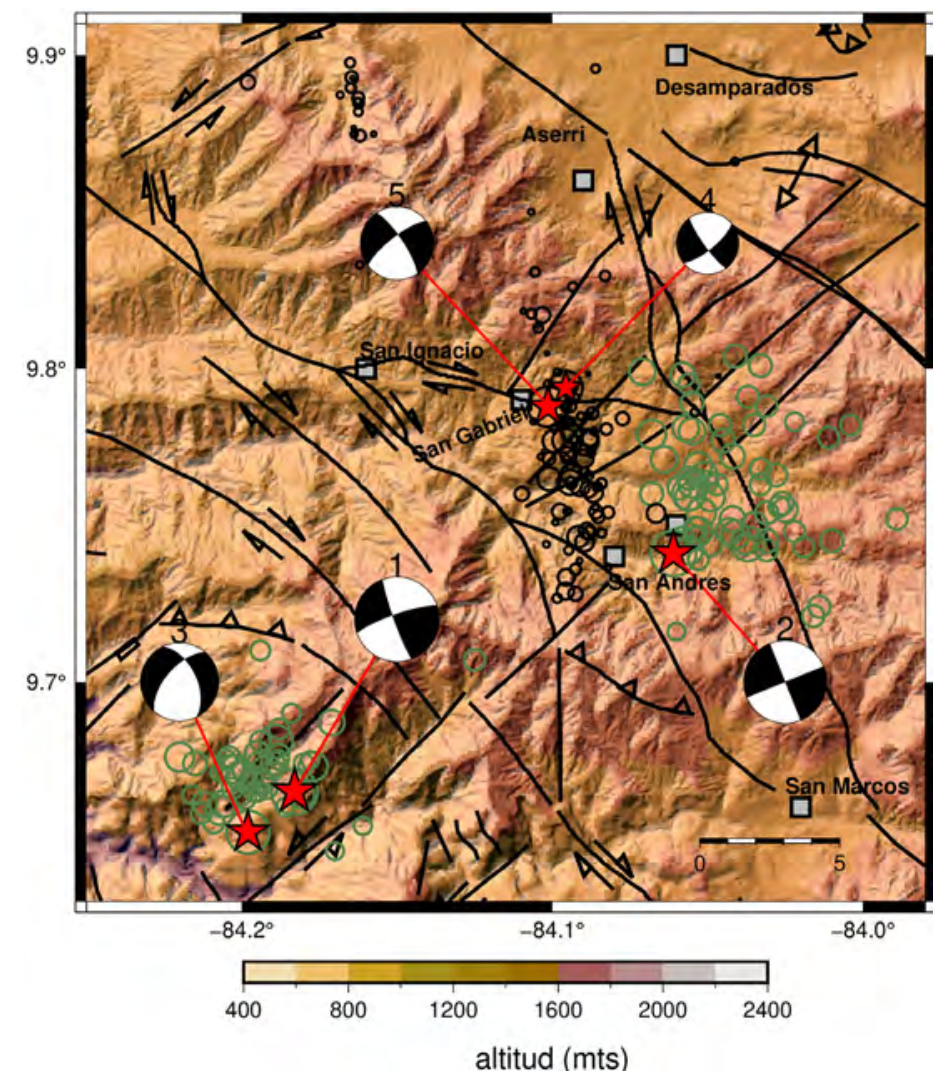

Figura 7. Los epicentros de la sismicidad anterior al 2018 están indicados por circunferencias verdes y los de ese año por circunferencias negras, todas proporcionales al tamaño de la magnitud. El número sobre la esfera focal representa: 1) el sismo de Altos El Aguacate (Mw 5,3), 2) el de Frailes (Mw 5,2), 3) el del 7 de agosto de 2014, (Ml 4,9), 4) el premonitor del 27 julio 2018 (Ml 3,9) y 5) el de San Gabriel (Ml 4,6). Los MF para los dos primeros fueron tomados de GCMT (2019) y los enumerados del tres al cinco se obtuvieron en este trabajo. 
Tabla 3. Parámetros sísmicos de los eventos mayores a Ml 4,0 en la región SW del Valle Central de Costa Rica a partir de 1989.

\begin{tabular}{|c|c|c|c|c|c|c|c|}
\hline Sismo & Fecha & $\begin{array}{l}\text { Magnitud } \\
\text { (Ml) }\end{array}$ & Ubicación & $\begin{array}{c}\text { Profundidad } \\
(\mathbf{k m})\end{array}$ & $\begin{array}{c}\text { IMM } \\
\text { máximo }\end{array}$ & $\begin{array}{c}\text { Tipo de } \\
\text { fallamiento }\end{array}$ & $\begin{array}{c}\text { Parámetros } \\
\text { strike/dip/rake }\end{array}$ \\
\hline $\begin{array}{c}\text { Alto El } \\
\text { Aguacate }\end{array}$ & $26 / 02 / 1989$ & 4,4 & $\begin{array}{c}\text { Latitud 9,665 } \\
\text { Longitud } \\
-84,183\end{array}$ & 21,5 & VI & $\begin{array}{c}\text { Transcurrente } \\
\text { sinestral }\end{array}$ & $251^{\circ} / 80^{\circ} / 6^{\circ}$ \\
\hline De Frailes & 09/08/1991 & 4,6 & $\begin{array}{c}\text { Latitud 9,741 } \\
\text { Longitud } \\
-84,061\end{array}$ & 10 & VII & $\begin{array}{c}\text { Transcurrente } \\
\text { sinestral }\end{array}$ & $158^{\circ} / 90^{\circ} /-180^{\circ}$ \\
\hline $\begin{array}{c}\text { Del } 7 \text { de } \\
\text { agosto de } \\
2014\end{array}$ & 07/08/2014 & 4,9 & $\begin{array}{c}\text { Latitud 9,652 } \\
\text { Longitud } \\
-84,198\end{array}$ & 22,6 & III & $\begin{array}{l}\text { Normal con } \\
\text { componente } \\
\text { SS sinestral }\end{array}$ & $221^{\circ} / 61^{\circ} /-41^{\circ}$ \\
\hline $\begin{array}{l}\text { De San } \\
\text { Gabriel }\end{array}$ & 27/07/2018 & 4,6 & $\begin{array}{l}\text { Latitud 9,788 } \\
\text { Longitud } \\
-84,101\end{array}$ & 15 & IV & $\begin{array}{c}\text { Transcurrente } \\
\text { dextral con } \\
\text { componente } \\
\text { normal }\end{array}$ & $326^{\circ} / 77^{\circ} /-165^{\circ}$ \\
\hline
\end{tabular}

\section{Discusión de resultados}

La deformación en la región central de Costa Rica es compleja, con fallas que muestran deslizamientos que se han interpretado como producto del desarrollo de un "sliver" de antearco con desplazamiento paralelo a la trinchera (Lewis et al., 2008; López, 2012) o bien que corresponde con el límite entre dos de los principales bloques de la corteza, la microplaca de Panamá y la placa Caribe (Kobayashi et al., 2014; Norabuena et al., 2004).

La relocalización de la sismicidad asociada al evento de San Gabriel indica que este fue superficial y con MF similar a otros que se han registrado en la región central del país. Por ejemplo, el terremoto de Cinchona de 2009 o el de Capellades en 2016 (Campos-Durán y QuinteroQuintero, 2020) que tuvieron cinemáticas parecidas.

El momento sísmico calculado para el sismo de San Gabriel fue de 6,533e+21 dinas-cm; lo cual corresponde a una ruptura de aproximadamente 1,4 km de longitud. El dato fue obtenido usando la relación $\log \mathrm{L}=0,279$ $\log$ (Mo) - 5,93 para Mo < 5 x 1025 dinas-cm de Shimazaki (1986); la cual, aunque fue derivada usando datos de Japón, proporciona una idea de la longitud del rompimiento.

La distribución epicentral de las réplicas indica una ruptura mayor, pero no siempre la relación entre el área de rompimiento del evento principal y la de estas últimas es de 1 (Ci Neo et al., 2021). Por lo que la fórmula empírica únicamente proporciona una idea del área, máxime que fue derivada para sismos de baja magnitud.
Wells y Coppersmith (1994) indican que la paleosismicidad y los estudios geológicos se pueden emplear para establecer parámetros de la fuente como ruptura, desplazamientos y segmentación; esto permitiría determinar una posible segmentación de la de Jaris, la cual con los datos disponibles es una buena candidata para ser la responsable del sismo de San Gabriel del 2018.

Parte de la sismicidad superficial $(\leq 25 \mathrm{~km})$ en la región central del país corresponde a sismos moderados o ligeros, como el de San Gabriel y para otros de mayor magnitud el periodo de recurrencia es mayor a 20 años, como fue mostrado por Quintero-Quintero y PorrasEspinoza (2018). El MF del evento en estudio es de corrimiento lateral con pequeña componente normal y está en concordancia con lo encontrado por Pacheco et al. (2006), quienes indican un régimen de esfuerzos transtensionales en toda la corteza de la placa continental en la zona central del país.

También se ha constatado que en un área aproximada de $2500 \mathrm{~km}^{2}$ dentro del CDCCR (Figura 1) se originan sismos asociados a fallamientos con dirección NE y de corrimiento lateral izquierdo, como el de Frailes de 1991, Altos El Aguacate de 1984, el de agosto de 2014 o el caso en estudio, donde las fallas presentan una dirección NNW o NW y son de corrimiento lateral derecho. López (2012) atribuye el régimen de esfuerzo actual en la zona central del país a la respuesta de la placa continental sobreyacente (Caribe) a los esfuerzos generados por la colisión de la placa Coco y a la relación colisión-indentación de la cordillera volcánica submarina del Coco en el Pacífico Sur de Costa Rica. 
Los eventos con mayor magnitud han ocurrido en fallas con dirección NE, como es el caso del sismo de Frailes de 1991, que Montero et al. (2016) lo asocian con la falla Navarro, ya que su epicentro, así como las de las réplicas se localizaron muy cerca de su traza. Estructuras como estas generalmente son de menor tamaño a las que tienen dirección NW paralelas a la MAT, como se muestra en la Figura 1.

\section{Conclusiones}

La sismicidad localizada entre el 20 de junio y 1 de agosto de 2018 en San Gabriel de Aserrí presentó profundidades entre los 8 y $15 \mathrm{~km}$, los mecanismos obtenidos usando inversión de ondas sísmicas y el impulso de los primeros arribos señalan un rumbo $\mathrm{N} 34^{\circ}-39^{\circ} \mathrm{W}$, lo que coincide con los epicentros de las réplicas ubicadas principalmente entre 0 y $7 \mathrm{~km}$ al SE del del sismo principal. Hacia el NW, la distribución fue más dispersa y fueron pocos los eventos localizados.

La magnitud de los sismos del 26 de febrero de 1989 (Ml 4,4), del 09 de agosto de 1991 (Ml 4,6) y del 27 de julio de 2018 (Ml 4,6) indican que las longitudes de los planos nodales asociados a las fallas no aflorantes que dieron origen a estos eventos son menores a 5,7 km.

Los sismos corticales se caracterizan por ser transtensionales con dirección de ejes de presión al NNE, subparalelos a la dirección de convergencia de las placas del Coco y Caribe y eje de extensión en sentido E-W en el antearco central de Costa Rica.

Los eventos ocurridos entre 1989 y 2018 han sido de débiles a fuertes, de desplazamiento lateral, tanto dextral como sinestral, prácticamente cortándose entre ellas en forma oblicua. En la escala de IMM registraron intensidades máximas de VII, algunos de ellos provocaron daños en estructuras como viviendas, carreteras y sistemas de agua potable y eléctrico, destacando los de Alto El Aguacate (1989) y Frailes (1991).

Las fallas conocidas y con control superficial muestran longitudes importantes que varían entre 70 y 85 km, tal como la Aguacaliente o La Candelaria, respectivamente; lo que es un indicador de un potencial importante para generar sismos fuertes, esto ha sido ratificado por eventos como el terremoto de Puriscal de 1990 o el de Frailes de 1991.

\section{Agradecimientos}

Al gobierno de Costa Rica por el financiamiento para la instalación y el mantenimiento de las estaciones sísmicas del OVSICORI-UNA, a través de la Ley $\mathrm{N}^{\circ}$ 8488. A la RSN-UCR y LIS-UCR por compartir datos con la red sísmica de la Universidad Nacional de Costa Rica. Al equipo de profesionales del OVSICORI-UNA por su arduo trabajo en mantener en operación la red sísmica y por el trabajo realizado para la recopilación de la sismicidad del país desde 1984. Al M.Sc. Hernán Porras por la revisión del texto y sus valiosos aportes. A los revisores, cuyos aportes y comentarios hicieron enriquecer el contenido técnico-científico de esta investigación. Esta investigación se llevó a cabo en el marco del proyecto 0178-18 de la Universidad Nacional de Costa Rica.

\section{Referencias}

Arias, O.; Denyer, P. (1991). Aspectos neotectónicos y geológicos de Puriscal y alrededores, Costa Rica. Revista Geológica de América Central, 12, 83-95. https://doi.org/10.15517/RGAC.V0I12.13046

Barquero, R.; Rojas, W.; Climent, A. (1989). El temblor del 26 de febrero de 1989: Informe sismológico y análisis preliminar de acelerogramas de la presa San Miguel. Instituto Costarricense de Electricidad.

Campos-Durán, D.; Quintero-Quintero, R. (2020). Intensidades macrosísmicas del sismo de Capellades del 30 de noviembre de 2016 (Mw = $5,4)$ y el contexto sísmico de la región central de Costa Rica. Boletín de Geología, 42(1), 57-68. https://doi.org/10.18273/revbol.v42n1-2020003

Carvalho, J.; Barros, L.; Zahradnik, J. (2019). Inversion for focal mechanisms using waveform envelopes and inaccurate velocity models: Examples from Brazil. Bulletin of the Seismological Society of America, 109(1), 138-151. https://doi. org/10.1785/0120180119

Ci Neo, J.; Huang, Y.; Yao, D.; Wei, S. (2021). Is the aftershock zone area a Good Proxy for the Mainshock Rupture Area? Bulletin of the Seismological Society of America, 111(1), 424438. https://doi.org/10.1785/0120190200 
Denyer, P.; Montero, W.; Alvarado, G.E. (2009). Atlas Tectónico de Costa Rica. Editorial Universidad de Costa Rica.

Fan, C.; Pavlis, G.; Tuncay, K. (2006). GCLgrid: A three-dimensional geographical curvilinear grid library for computational seismology. Computers \& Geosciences, 32(3), 371-381. https://doi. org/10.1016/j.cageo.2005.07.001

Fernández, M.; Rojas, W. (2001). Amenaza sísmica y tsunamis en el territorio de Costa Rica. Editorial Universidad de Costa Rica.

GCMT. (2019). Global CMT Catalog Search. Global Centroid Moment Tensor. http://www.globalcmt. org/CMTsearch.html

Güendel, F. (1989). Secuencia sísmica de El Alto del Aguacate, febrero y marzo de 1989. OVSICORIUNA, Catálogo de Temblores 1989, 155161.

Havskov, J.; Ottemoller, L. (1999). SeisAn Earthquake analysis software. Seismological Research Letters, 70(5), 532-534. https://doi.org/10.1785/ gssrl.70.5.532

Kobayashi, D.; LaFemina, P.; Geirsson, H.; Chichaco, E.; Abrego, A.; Mora, H.; Camacho, E. (2014). Kinematics of the western Caribbean: Collision of the Cocos Ridge and upper plate deformation. Geochemistry, Geophysics, Geosystems, 15(5), 1671-1683. https://doi. org/10.1002/2014GC005234

Lewis, C.; Boozer, C.; López, A.; Montero, W. (2008). Collision versus sliver transport in the hanging wall at the Middle America subduction zone: Constraints from background seismicity in central Costa Rica. Geochemistry, Geophysics, Geosystems, 9(7). https://doi. org/10.1029/2007GC001711

López, A. (2012). Andersonian and Coulomb stresses in Central Costa Rica and its fault slip tendency potential: new insights into their associated seismic hazard. Geological Society, London, Special Publications, 367, 19-38. https://doi. org/10.1144/SP367.3

Marshall, J.; Fisher, M.; Gardner, T. (2000). Central Costa Rica deformed belt: kinematics of diffuse faulting across the western Panama block. Tectonics, 19(3), 468-492. https://doi. org/10.1029/1999TC001136

Montero, W.; Alvarado, G.E. (1995). El terremoto de Patillos del 30 de diciembre de $1952(\mathrm{Ms}=5,9)$ y el contexto neotectónico de la región del volcán Irazú, Costa Rica. Revista Geológica de América Central, 18, 25-42. https://doi.org/10.15517/rgac. v0i18.13522

Montero, W. (1999). El terremoto del 4 de marzo de 1924 (Ms 7,0): ¿Un gran temblor intraplaca relacionado al límite incipiente entre la placa Caribe y la microplaca Panamá? Revista Geológica de América Central, 22, 25-62. https:// doi.org/10.15517/RGAC.V0I22.8586

Montero, W.; Rojas, W. (2014). Las fallas Purires y Picagres, y su relación con la secuencia sísmica del Puriscal de 1990. Revista Geológica de América Central, 50, 39-69. https://doi.org/10.15517/ RGAC.V0I50.15107

Montero, W.; Linkimer, L.; Rojas, W. (2016). El sistema de Fallas Navarro: desplazamientos izquierdos a lo largo del cinturón deformado del centro de Costa Rica. Revista Geológica de América Central, 55, 71-100. https://doi.org/10.15517/ RGAC.V55I0.27062

Norabuena, E.; Dixon, T.; Schwartz, S.; DeShon, H.; Newman, A.; Protti, M.; González, V.; Doramn, L.; Flueh, E.; Lundgren, P.; Pollitz, F.; Sampson, D. (2004). Geodetic and seismic constraints on some seismogenic zone processes in Costa Rica. Journal of Geophysical Research: Solid Earth, 109(B11). https://doi.org/10.1029/2003JB002931

Pacheco, J.; Quintero, R.; Vega, F.; Segura, J.; Jiménez, W.; González, V. (2006). The Mw 6.4 Damas, Costa Rica, earthquake of 20 November 2004: Aftershocks and slip distribution. Bulletin of the Seismological Society of America, 96(4A), 13321343. https://doi.org/10.1785/0120050261

Rojas, W.; Lindholm, C.; Bungum, H.; Boschini, L.; Climent, A.; Barquero, R.; Alvarado, G.; Soto, G.; Montero, W.; Fernández, M.; Protti, M.; Moya, A.; Esquivel, L.; Schmidt, V. (1998). Seismic hazard analysis for the Metropolitan Area of the Central Valley, Costa Rica. Technical Report, NORSAR, Norway. 
RSN-UCR. (2014). Informe del sismo 7 de agosto del 2014, 2:50 am., Mag: 4,6 Mw, SENTIDO. Red Sismológica Nacional de la Universidad de Costa Rica. https://rsn.ucr.ac.cr/actividad-sismica/ ultimos-sismos/2285-sismo-07-de-agosto-del2014-2-50-am-mw-4-6

RSN-UCR. (2015). Terremoto de Frailes, 9 de agosto de 1991. Red sismológica Nacional de la Universidad de Costa Rica. https://rsn.ucr. ac.cr/documentos/educativos/vulcanologia/26sismologia/sismos-historicos/3236-terremoto-defrailes-9-de-agosto-de-1991

Shimazaki, K. (1986). Small and large earthquakes: The effects of the thickness of seismogenic layer and the free surface. In: S. Das, J. Boatwright, C. Scholz (eds.). Earthquake Source Mechanics (pp. 209-216). Vol. 37. American Geophysical Union. https://doi.org/10.1029/GM037p0209

Sokos, E.; Zahradnik, J. (2008). ISOLA a Fortran code and a Matlab GUI to perform multiple-point source inversion of seismic data. Computers and Geosciences, 34(8), 967-977. https://doi. org/10.1016/j.cageo.2007.07.005

Sokos, E.; Zahradnik, J. (2013). Evaluating centroid-moment-tensor uncertainty in the new version of ISOLA software. Seismological Research Letters, 84(4), 656-665. https://doi. org/10.1785/0220130002

Quintero-Quintero, R.; Porras-Espinoza, H. (2018). Recurrencia de sismos en el Valle Central de Costa Rica. Revista Geográfica de América Central, 4(61E), 63-80. https://doi.org/10.15359/ rgac.61-4.3
Quintero, R.; Kissling, E. (2001). An improved P-wave velocity reference model for Costa Rica. Geofísica Internacional, 40(1), 3-19.

UNAVCO. (2020). Plate Motion Calculator. https:// www.unavco.org/software/geodetic-utilities/ plate-motion-calculator/plate-motion-calculator. html\#references

Wald, D.; Worden, B.; Quitoriano, V.; Pankow, K. (2006). ShakeMap manual: technical manual, user's guide, and software guide. U.S. Geological Survey.

Wells, D.; Coppersmith, K. (1994). New empirical relationship among magnitude, rupture length, rupture width, rupture area, and surface displacement. Bulletin of the Seismological Society of America, 84(4), 974-1002.

Wessel, P.; Luis, J.F.; Uieda, L.; Scharroo, R.; Wobbe, F.; Smith, W.H.F.; Tian, D. (2019). The Generic Mapping Tools version 6. Geochemistry, Geophysics, Geosystems, 20(11), 5556-5564. https://doi.org/10.1029/2019GC008515

Zahradnik, J.; Sokos, E. (2018). Fitting waveform envelopes to derive focal mechanisms of moderate earthquakes. Seismological Research Letters, 89(3), 1137-1145. https://doi. org/10.1785/0220170161

Zimmermann, B. (2009). Earthquake Focal Mechanism. Wolfram Demonstrations Project. http://demonstrations.wolfram.com/ EarthquakeFocalMechanism/

Fecha de recibido: 09 de octubre de 2020 Fecha de aprobado: 02 de agosto de 2021 